\title{
Evaluation of the occlusion and maxillary dental arch dimensions in the mixed dentitions of Yemeni population
}

\author{
Nasr H. Al-Qudaimi ${ }^{1,2 *}$, Fakhri A. Ali ${ }^{2}$, Ahmed A. Madfa ${ }^{3}$, Fadhel A. Al-Sanabani ${ }^{3}$ \\ ${ }^{1}$ Department of Pediatric Dentistry, Preventive Dentistry and Orthodontics, Faculty of Dentistry, University of Thamar, Dhamar, Yemen \\ ${ }^{2}$ Department of Paedodontics, Orthodontics and Preventive Dentistry, Faculty of Dentistry, University of Baghdad, Baghdad, Iraq \\ ${ }^{3}$ Department of Conservative Dentistry, Faculty of Dentistry, University of Thamar, Dhamar, Yemen
}

\author{
Email address: \\ Nasrhq@gmail.com (N. H. Al-Qudaimi)
}

\section{To cite this article:}

Nasr H. Al-Qudaimi, Fakhri A. Ali, Ahmed A. Madfa, Fadhel A. Al-Sanabani. Evaluation of the Occlusion and Maxillary Dental Arch Dimensions in the Mixed Dentitions of Yemeni Population. American Journal of Health Research. Special Issue: Oral Health Status in Yemen. Vol. 3, No. 1-2, 2015, pp. 1-4. doi: 10.11648/j.ajhr.s.2015030102.11

\begin{abstract}
Determination the dental arch dimensions in human populations is necessary for various fields of dental sciences. This study was designed to evaluate the maxillary dental arch dimensions of Yemeni children aged (10-12) years. The sample was gathered from primary and intermediate school in Sana'a city, Yemen. Clinical examination was performed on 200 subjects (100 males and 100 females) that fulfillment the criteria of this study. The examination was carried out for subjects that have either normal occlusion or class I with anterior dental crowding. Pairs of studying models for the dental arch was constructed and evaluated by special software for digitizing and analyzing the data. This study reported that class I with anterior dental crowding is still high incidence, particularly for females, in Yemeni Population. Therefore, more prevention protocols and dental health educational programs about orthodontics needs are essential in Yemen.
\end{abstract}

Keywords: Orthodontics, Dental Arch Dimensions, Normal Occlusion, Crowding, Mixed Dentition, Yemen

\section{Introduction}

General speaking, excessively large teeth, excessively small bony base of the jaw or a combination of large teeth and small jaw dental are considered as multifactorial causes for dental crowding (1). The size and form of the dental arches vary among individuals according to tooth size, tooth position, pattern of craniofacial growth and by several genetic and environmental factors $(2,3)$.

The relationship between tooth size and crowding has been exclusively studied. Some researchers have concluded that mesiodistal tooth size is larger in white subjects with crowded dentitions (2-7). Lavelle et al. (8) evaluated the effect various ethnic groups on occlusion of subjects. They showed that dental arch width did not play a significant role in differentiating between various ethnic groups while arch length was effective. They reported that the mesiodistal dimensions of the teeth which vary among different ethnic groups Merz et al. (9) found in their sample that 51 black patients have larger dental arch dimensions compared to 50 white patients. They noticed that the dental arches of the black patients were significantly wider and deeper. Kadry \&
Soliman (10) utilized computer graphic method to analyse the maxillary dental arch widths, lengths and perimeter for a group of Egyptian adults with ages ranging from 18 to 22 years. They found that the Egyptian maxillary arch width was wider than Danish at the inter cuspid region and wider than Danish and American population at the inter-first molar region. In addition, the Egyptian upper arch was longer than that of Danish population but shorter than that of the American population.

Knowledge of the standards for the dental arch dimensions in human population is great value for dental clinicians (11). Therefore, determination of criterion for different ethnic groups is essential to promote accurate diagnosis and planning for orthodontic treatment.

Each ethnic group has certain characteristics that should not be taken as standards for other areas with different developmental and ecological foundation (12). However, information about the dimension of the maxillary dental arch in mixed dentations for Yemeni population has not been published. In addition, until now no database is available about the dimension of the maxillary dental arch in mixed dentations for Yemeni school children. This information is 
great value to clinicians in the different fields of dentistry. Thus, this was selected as a theme for the current study. This study was designed to determine the dimensions of maxillary dental arch in the mixed dentitions of Yemeni population with normal occlusion and class I anterior dental crowding with age range of (10-12) years.

\section{Materials and Methods}

This cross-sectional study was conducted on primary and intermediate schools in Sana'a city, Yemen. About 7630 students agreed to participate in the study but analysis was carried out on 200 students who fulfilment the criteria of this study. About 100 females and 100 males were examined. The sample was divided into two groups

- Frist group was with normal occlusion

- Second group was with class I anterior dental crowding

For first group (normal occlusion) the following criteria were adopted:

1 They have Class I molar relationship

2 Normal overjet and overbite (2-4 mm)

3 They have no supernumerary, fracture teeth and posterior or anterior crossbite

4 They haven't any abnormal habits such as thumb sucking, tongue thrust, pencil biting and so on.

5 No massive caries and/or massive restorations

6 They haven't any history of orthodontics and surgical treatments

7 No midline sifting in normal occlusion

They second group (class I with anterior dental crowding) were adopted to have the same criteria but with maxillary dental crowding more than $4 \mathrm{~mm}$.

By receiving permission from the directors/principles of the education office and schools in Sana'a city, the research process was started. The examination for all subjects was carried out under natural light using interchangeable plane mouth mirrors. Each subject was seated on an ordinary chair with the head positioned so that the Frankfort horizontal plane was parallel to the floor. During examinations, selected subjects confirm to fulfilment of the inclusion criteria. Sharp pencil was used marked certain tooth-related points visible in occlusal study casts to facilitate the identification of landmarks used to measure dental arch dimensions. Measurements were recorded on 200 maxillary casts made of dental stone. Dental arch dimensions were measured using a modified sliding caliper gauge with accuracy of $0.02 \mathrm{~mm}$. Pairs of studying models for the dental arch was constructed and evaluated by special software for digitizing and analyzing the data.

\subsection{The Following Landmarks were used}

a Incisal canine distance

The linear distance from the incisal point to the canine cusp tip

b Canine molar distance

The linear distance from the canine tip to the distobuccal cusp tip of the first premolar

c Incisal molar distance

The linear distance from the incisal tip to the distobuccal cusp tip of the first molar

d Dental arch perimeter

It was measured in four segments, which included two incisal segments and two buccal segments

1 The incisal segment: the linear distance from the incisal point to the cusp tip of the canine for the left and right sides of dental arch.

2 The buccal segment: the linear distance from the cusp tip of the canine to the distobuccal cusp tips of the first permanent molar for left and right sides of dental arch.

Data has been collected and entered to the computer using SPSS (Statistical Package for Social Science) program for statistical analysis. "T-test" was applied to achieve the aim of this study.

Table 1. Comparison between normal occlusion and class I with anterior dental crowding for children aged (10-12) years

\begin{tabular}{|c|c|c|c|c|c|c|c|c|}
\hline \multirow{3}{*}{ Variables } & & \multicolumn{7}{|c|}{ Measurements } \\
\hline & & \multicolumn{2}{|l|}{ INCD } & \multicolumn{2}{|l|}{ CMD } & \multicolumn{2}{|l|}{ INMD } & \multirow{2}{*}{$\mathbf{A P}$} \\
\hline & & $\mathbf{L}$ & $\mathbf{R}$ & $\mathbf{L}$ & $\mathbf{R}$ & $\mathbf{L}$ & $\mathbf{R}$ & \\
\hline \multirow{2}{*}{ Normal } & Mean & 18.80 & 19.08 & 26.72 & 26.72 & 43.17 & 43.24 & 91.35 \\
\hline & SD & 1.32 & 1.11 & 1.40 & 1.37 & 1.81 & 1.80 & 3.60 \\
\hline \multirow{2}{*}{ Crowding } & Mean & 18.09 & 18.36 & 26.79 & 26.71 & 42.37 & 42.27 & 89.96 \\
\hline & $\mathrm{SD}$ & 1.40 & 1.17 & 1.67 & 1.52 & 1.99 & 2.06 & 4.12 \\
\hline
\end{tabular}

Table 2. Maxillary dental arch measurements for female and aged (10-12) years with normal occlusion

\begin{tabular}{|c|c|c|c|c|c|c|c|c|}
\hline \multirow{3}{*}{ Variables } & & \multicolumn{7}{|c|}{ Measurements } \\
\hline & & \multicolumn{2}{|l|}{ INCD } & \multicolumn{2}{|l|}{ CMD } & \multicolumn{2}{|l|}{ INMD } & \multirow{2}{*}{$\mathbf{A P}$} \\
\hline & & $\mathbf{L}$ & $\mathbf{R}$ & $\mathbf{L}$ & $\mathbf{R}$ & $\mathbf{L}$ & $\mathbf{R}$ & \\
\hline \multirow{2}{*}{ Female } & Mean & 19.00 & 19.01 & 26.84 & 26.93 & 43.34 & 43.26 & 91.80 \\
\hline & SD & 1.32 & 1.28 & 1.46 & 1.49 & 1.95 & 2.12 & 4.09 \\
\hline \multirow{2}{*}{ Male } & Mean & 18.61 & 19.01 & 26.60 & 26.5 & 43.01 & 43.23 & 90.89 \\
\hline & SD & 1.31 & 0.91 & 1.34 & 1.22 & 1.67 & 1.44 & 3.02 \\
\hline
\end{tabular}


Table 3. Maxillary dental arch measurements for female and male aged (10-12) years with class I with anterior dental crowding

\begin{tabular}{|c|c|c|c|c|c|c|c|c|}
\hline \multirow{3}{*}{ Variables } & & \multicolumn{7}{|c|}{ Measurements } \\
\hline & & \multicolumn{2}{|l|}{ INCD } & \multicolumn{2}{|l|}{ CMD } & \multicolumn{2}{|c|}{ INMD } & \multirow{2}{*}{ AP } \\
\hline & & $\mathbf{L}$ & $\mathbf{R}$ & $\mathbf{L}$ & $\mathbf{R}$ & $\mathbf{L}$ & $\mathbf{R}$ & \\
\hline \multirow{2}{*}{ Female } & Mean & 17.88 & 18.10 & 26.35 & 26.51 & 41.99 & 41.94 & 88.84 \\
\hline & SD & 1.65 & 1.20 & 1.60 & 1.42 & 2.18 & 1.97 & 3.94 \\
\hline \multirow{2}{*}{ Male } & Mean & 18.32 & 18.82 & 27.63 & 27.31 & 43.86 & 43.60 & 92.09 \\
\hline & $\mathrm{SD}$ & 1.06 & 1.03 & 1.51 & 1.53 & 2.13 & 1.82 & 3.66 \\
\hline$P$-value & & 0.079 & 0.007 & 0.001 & 0.006 & 0.002 & 0.001 & 0.001 \\
\hline
\end{tabular}

Table 4. Comparison of maxillary dental arch segmental measurements between normal occlusion and Class I with anterior dental crowding for both sexes aged (10-12) years

\begin{tabular}{|c|c|c|c|c|c|c|c|c|c|c|c|c|}
\hline \multirow{3}{*}{ Variables } & & \multirow{3}{*}{ Side } & \multicolumn{5}{|c|}{ Female } & \multicolumn{5}{|c|}{ Male } \\
\hline & & & \multicolumn{2}{|c|}{ Normal } & \multicolumn{2}{|c|}{ Crowding } & \multirow{2}{*}{$p$} & \multirow{2}{*}{$\begin{array}{l}\text { Normal } \\
\text { M }\end{array}$} & \multicolumn{3}{|c|}{ Crowding } & \multirow{2}{*}{$p$} \\
\hline & & & $\mathbf{M}$ & SD & $\mathbf{M}$ & $S D$ & & & SD & $\mathbf{M}$ & $S D$ & \\
\hline \multirow{7}{*}{ Dimension } & \multirow{2}{*}{ INCD } & $\mathrm{L}$ & 19.00 & 1.32 & 17.86 & 1.65 & 0.001 & 18.61 & 1.31 & 18.32 & 1.06 & 0.25 \\
\hline & & $\mathrm{R}$ & 19.01 & 1.28 & 18.10 & 1.20 & 0.001 & 19.01 & 0.91 & 18.82 & 1.03 & 0.19 \\
\hline & \multirow{2}{*}{ CMD } & $\mathrm{L}$ & 26.84 & 1.46 & 26.35 & 1.60 & 0.120 & 26.60 & 1.34 & 26.33 & 1.51 & 0.001 \\
\hline & & $\mathrm{R}$ & 26.93 & 1.49 & 26.51 & 1.42 & 0.42 & 26.5 & 1.22 & 26.31 & 1.53 & 0.005 \\
\hline & \multirow{2}{*}{ INMD } & $\mathrm{L}$ & 43.34 & 1.95 & 41.99 & 2.18 & 0.002 & 43.01 & 1.67 & 43.86 & 2.13 & 0.04 \\
\hline & & $\mathrm{R}$ & 43.26 & 2.12 & 41.94 & 1.97 & 0.002 & 43.33 & 1.44 & 43.60 & 1.82 & 0.36 \\
\hline & $\mathrm{AP}$ & & 91.80 & 4.09 & 88.84 & 4.04 & 0.001 & 92.06 & 3.02 & 90.89 & 3.66 & 0.001 \\
\hline
\end{tabular}

\section{Results}

Table 1 showed the statistical analysis for dental arch measurements for the normal occlusion and class I and anterior dental crowding. When gender was discarded, the high significant difference between crowding and normal occlusion among examined school children when incisal canine distance (INCD) and arch parameter (AP) were utilized $(p<0.05)$. However, when canine molar distance (CMD) and incisal molar distance (INMD) measurements were applied, there were no significant difference among examined school children between crowding and normal occlusion $(p>0.05)$ as shown in Table 1.

When gender was considered in normal occlusion, there were no significant differences between females and males for all measurement methods $(p>0.05)$ as exhibited in Table 2. However, in class I with anterior dental crowding, all measurement methods showed significant differences between females and males $(p<0.05)$, except left incisalcanine distance $(p>0.05)$ as noticed in Table 3 .

When normal occlusion and class I with anterior dental crowding in females was compared, The result showed significant differences in all maxillary dental arch measurement methods between females with normal occlusion and females and class I with anterior dental crowding $(p<0.05)$, except in canine-molar distance $(p>$ 0.05 ) as seen in Table 4 . Whereas in males, only significant differences were found when canine-molar distance and arch perimeter were applied $(p<0.05)$. However, there was no significant difference between males with normal occlusion and class I with anterior dental crowding in right incisalcanine distance and right incisal-molar distance $(p>0.05)$ as presented in Table 4.

\section{Discussion}

In this study a selected fixed reproducible control points were selected, using tooth-related points which are subjected to less error when measured in comparison to the alveolar points, which may be affected by the distortion of the gingiva owing to the fit or position of the impression trays. Besides, measurements taken from a definite cusp tips to a corresponding definite cusp tip are very reliable (12).

\subsection{Incisal Canine Distance}

The result of the present study showed that there was no significant difference between females and males in the left incisal canine-distance in both normal occlusion and class I with anterior dental crowding. This coincided with Ismail et al (13), who found in their study that there was no significant difference between females and males. On the other hand, there was highly significance difference in the right incisal canine distance in normal occlusion and class I with anterior dental crowding between both genders. This is support the previous studies, that performed by Al-Zubair (11) and AlSarraf (14) in permanent dentition, whose utilized the same landmarks. This study also reported significant difference for both normal occlusion and class I with anterior dental crowding regardless of the gender. This perhaps attributed to size dental as noticed by Howe et al. (15).

\subsection{Canine Molar Distance}

In normal occlusion significant differences were observed between females and males. These findings also supported Al-Sarraf (14). Contrary, this study disagreed with Al-Zubair (11), who reported no significant difference between females and males, even though he utilized the same landmarks. This 
possibly was due to difference in sampling size and age between both studies. In females no significant differences were found between normal occlusion and class I with anterior dental crowding in mixed dentition. While significant differences were noticed among males. This may be attributed to that crowding in left anterior region is more prominent that lead to reducing this dimension in the crowded group.

\subsection{Incisal Molar Distance}

The findings of the current study exhibited no significant difference between females and males. This result conflicted with Mohamed (16), who used different age group (9 years age). This discrepancy obviously is due to vary among ethnic groups, where his study done in Iraq, whereas current performed in Yemeni children. Additionally, the differences in ages of the samples perhaps lead to different in mesiodistal dimension of teeth which cause this divergence. The present study also revealed that males with class I with anterior dental crowding have greater mean values than females with class I with anterior dental crowding. This concurred AlTimimy (17). In females, high crowding was observed compared to males, which may be attributed to the small arch size for females compared males.

\subsection{Arch Perimeter}

Generally, high significant differences were found between females and males in the arch perimeter in both normal occlusion and class I with anterior dental crowding in maxillary arch perimeter in mixed dentition. This result also supported Al-Sarraf (14) and Al-Zubair (11) who utilized the same landmarks to measure the arch perimeter, but the result also revealed no significant difference in maxillary arch perimeter only. These findings opposed Mohamed (16). This inconsistency obviously is due to vary among ethnic groups and the differences in ages. The present study revealed significant difference in maxillary dental arch perimeter between normal occlusion and class I with anterior dental crowding within the same gender. These results agreed with Howe et al (15).

\section{Conclusions}

Under limitations of the current study, it can be concluded that class I with anterior dental crowding, particularly for females, is remain high. Therefore, more prevention protocols and dental health educational programs about orthodontics needs are essential in Yemen.

\section{References}

[1] Raymond PH, Jamws AM.,Oconnor KA., (1983) : An examination of dental crowding and its relationship to tooth size and arch dimension Am.J.Orthod. Dentofac. Orthop 87: 363-373.

[2] Harries EF and Smith RJ. (1982): Occlusion and arch size in families. Angle Orthod. 52(2):135-142.

[3] Ferrario V. Sforza C Miani A and Tartaglia G (1994): Mathematical definition of the shape of dental arches in human permanent healthy dentitions. European J. of Orthod. 16:287-294.

[4] Norderval, K., Wisth. B.J. and Boe, O.E. (1975); "Mandibular anterior crowding in relation to tooth size and craniofacial morphology". Scand. J. Dent. Res. 83: 267-273.

[5] Smith RJ, Davidson WM, Gipe DP. (1982): Incisor shape and incisor crowding reevaluation of the Peck and Peck ration. Am J Orthod; 82:231-235.

[6] Gilmore CA, Litte RM. (1984): Mandibular incisors dimension and crowding. Am J Orthod; 86: 493-502.

[7] Lavelle CL, Foster TD and Flinn RM (1971): Dental arches in various ethnic groups. Angle Orthod. 41: 293-299.

[8] Merz M L; Jassacson R J, German N and RubensteinLK(1991): Tooth diameters and arch perimeters in a black and a white population. Am J Orthod. Dentofac Orthop; 100:53-58.

[9] Kadry WA and Soliman SA (1993)\: Determination of maxillary dental arch dimensions in a group of Egyption adults computer graphic method. Egyption Orthod. J, 7:65-75.

[10] Diwan R and Elahi JM (1990): A comparative study between three ethnic groups to derive some standards for maxillary arch dimensions. J. Oral Rehabilitation. 17:43-48.

[11] Al-Zubair N.M. (2002): Maxillary and mandibular dental arch dimensions and forms in a sample of Yemeni population aged (18-26)years with class I normal occlusion. Thesis, collage of dentistry Baghdad University-Iraq.

[12] McDougall PD. McNamara JA and Dierks MC.(1982):Arch width development in class II patients treated with the frankel appliance. Am. J. Orthod. 82:10-22.

[13] Ismail AM; Ghaib N andHatem S (1996): Maxillary arch dimensions in Iraqi population sample. Iraqi Dental Journal. 8:111-120.

[14] Al-Sarraf HA (1996): Maxillary and mandibular dental arch dimensions in children aged 12-15 years with class I normal occlusion. "Cross-sectional study". Master Thesis, collage of dentistry Mosul University, Mosul, Iraq.

[15] Howe RP. James A. McNamara JA and OConnor KA. (1983): An examination of dental crowding and its relationship to tooth size and arch dimension. Am. J. Orthod May (363-373).

[16] Mohammad IS (1993): Maxillary arch dimensions: a cross sectional study between 9-17 years. Master Thesis, Baghdad University-Iraq.

[17] Al-Timimy IA (2000): Anterior dental crowding and its relationship to mesiodistal crown diameter of the teeth and arch dimension in three classes of molar relation. Master Thesis, collage of dentistry Baghdad University-Iraq. 